\title{
Health Seeking Behavior and Associated Factors among Individuals with Cough in Yiwu, China: A Population-based Study
}

xiaoyan sun

Yiwu Center for Disease Control and Prevention

Shuying Luo

Yiwu Center for Disease Control and Prevention

Lingqiao Lou

Yiwu Center for disease control and prevention

Hang Cheng

Yiwu center for disease control and prevention

Zhen Ye

Centers for Disease Control and Prevention

Jianwei Jia

Yiwu center for disease control and prevention

Yina Wei

Yiwu center for disease control and prevention

Jingbo Tao

Yiwu center for disease control and prevention

Hanqing He ( $\nabla$ hanqinghe@cdc.zj.cn )

Zhejiang provincial Center for Disease Control and Prevention, Hangzhou https://orcid.org/0000-00030863-5177

Research article

Keywords: Cough, health seeking decision, choice of medical facility

Posted Date: October 13th, 2020

DOI: https://doi.org/10.21203/rs.3.rs-89505/v1

License: (c) (i) This work is licensed under a Creative Commons Attribution 4.0 International License.

Read Full License 


\section{Abstract}

Background: Previous studies have shown that a certain proportion of the population did not seek medical treatment after coughing, and understanding the potential reasons is crucial for disease prevention and control.

Method: A population-based study was conducted with the probability proportional to population size sampling in Yiwu, Zhejiang, China. A total of 5,855 individuals aged $\geq 15$ years lived in Yiwu, China for more than 6 months were included. All participants completed a pad-based questionnaire to collect detailed information by a face-to-face interview. Characteristics of individuals were described by categories of health seeking behavior using frequency and percentage. Univariate and multivariate logistic regression analyses were performed to estimate the associations of social-demographic and cough characteristics with health seeking behavior.

Results: $19.3 \%(1,129 / 5,855)$ of participants had a cough in the past month, $40 \%(452 / 1,129)$ had sought medical treatment. Of these, $26.5 \%(120 / 452)$ chose hospitals at county level or above. Individuals aged $\geq 65$ years old $(\mathrm{OR}=2.25,95 \% \mathrm{Cl}: 1.23,4.12)$, female $(\mathrm{OR}=1.57,95 \% \mathrm{Cl}: 1.21,2.06)$, living in rural areas $(\mathrm{OR}=1.30,95 \% \mathrm{Cl}: 1.003,1.69)$, persistent cough for $3-8$ weeks (OR=2.91, 95\% Cl: 1.72, 4.92) and with more accompanying symptoms $\left(P_{\text {trend }}<0.001\right)$ were more likely to seek medical treatment, but those coughed for $>8$ weeks were not $(p>0.5)$. Female $(\mathrm{OR}=0.33,95 \% \mathrm{Cl}: 0.21,0.54)$ and people living in rural areas $(\mathrm{OR}=0.57,95 \% \mathrm{Cl}: 0.36,0.92)$ were less likely to choose hospitals at county level or above while the higher educated were more likely to $(\mathrm{OR}=3.29,95 \% \mathrm{Cl}: 1.35,8.02)$. Those who coughed for more than 2 weeks were more likely to choose hospitals at or above the county level. But the number of accompanying symptoms does not show any significant relationship with the choice of medical facility.

Conclusion: The present study found that age, gender, living areas and features of cough were associated with health seeking behavior. It is worth noting that coughing too long(e.g. >8 weeks) instead of seeking medical treatment. Targeted measures should be developed based on the key factors found in this study to guide persons to seek medical treatment more scientifically.

\section{Background}

Cough is an essential defensive mechanism. It is not only a common symptom of respiratory diseases but also can reflect significant diseases ${ }^{[1,2]}$. More importantly, cough is an important factor in the spread of respiratory infectious diseases ${ }^{[3,4]}$. According to the International Standards for Tuberculosis Care, all persons with otherwise unexplained productive cough lasting 2-3 weeks or more should be evaluated for tuberculosis ${ }^{[5,6]}$. Recent studies also found that pertussis characterized by persistent cough is more prevalent in some areas, with a prevalence of $10.9-24.5 \%{ }^{[7-9]}$. In China, the prevalence of cough fluctuates between $9-64 \%$, and the corresponding burden of disease cannot be underestimated ${ }^{[10]}$. Thus scientific health seeking behavior of patients with cough is of great importance, which is conducive to the 
early diagnosis and treatment of diseases, especially contribute to the promptly prevention and control of respiratory infectious diseases.

However, previous studies found that a high proportion of patients who did not seek medical treatment after cough, and socio-demographic charactersistics were associated with health-seeking decision [11-13]. However, most of them were conducted abroad and found that evidence describing the potential risk factors are heterogeneous. A risk factor in one setting may promote the health-seeking decision but not in other settings. For example, a Ethiopia study found that higher income group were more likely to seek medical service ${ }^{[14]}$, while studies in South African ${ }^{[12]}$ and Zambia ${ }^{[15]}$ showed an opposite association for the same risk factor, and study in India ${ }^{[16]}$ found there was no correlation between income and healthseeking decision. The same situation occurs in other risk factors, such as age, gender, occupation and smoking status ${ }^{[13,15,17-19]}$. Besides, some factors were studied extensively in many studies (namely age, gender, education, occupation and income) while others may only appear in very few studies (namely, features of cough ${ }^{[13,20]}$. What'more, there is still no study has focused on the association between the duration of a cough and health seeking behavior.

Besides, studies on the choice of medical facilities for individuals with cough is very limited. In China, only very few studies have conducted at health facility level and focused on the individuals with presumptive tuberculosis ${ }^{[21-23]}$.

Here, a community survey provided us with a unique opportunity to look at the health seeking behavior of individuals with cough. The aim of the present study was to describe the health seeking behaviors (including health-seeking decision and choice of medical facility) of individuals with cough, and to examine the associations of social-demographic characteristics and features of cough with health seeking behavior.

\section{Methods}

\section{Study population}

All individuals aged $\geq 15$ years and were living in Yiwu for more than 6 months were considered as the source population. 50 clusters were selected from 14 town/streets in Yiwu with the probability proportional to population size sampling method, followed by a quota sampling method based on age and sex to select 110 individuals from each cluster. Then well-trained investigators went to each cluster to interview individuals who met the above criteria through simple random sampling.

All individuals completed a pad-based questionnaire to collect detailed information by a face-to-face interview, including socio-demographic characteristics, features of cough and health seeking behaviors. Among them, individuals who have had a cough in the past month were included in the present study. 


\section{Measurement}

\section{Assessment of health seeking behavior}

Based on the availability of data, the present study defined health seeking behavior as health-seeking decision and choice of medical facility. Participants were asked "Did you go to a medical facility for treatment after your last cough?" to acquire their health-seeking decision. If the participants answered "Yes", they were further asked "which medical facility did you choose". Options include clinics, 14 township hospitals or community health service centers, municipal hospitals, and others (open answer). We categorized the choice of medical facility into hospitals at county level or above and community health service center or below.

\section{Other covariates}

Cough was assessed by asking participants "Have you had a cough during the past month". If the participants answered "Yes", they were further asked the cough duration and whether they had accompanying symptoms, such as fever, expectoration, sore throat, runny nose, dyspnea, headache, fatigue, lethargy etc. We categorized the duration of cough period into $\leq 2$ weeks, 3-8 weeks and $>8$ weeks, and defined $\leq 2$ weeks as the reference group. And numbers of accompanying symptoms into 3 groups, namely 0 (reference), 1, 2 and above.

Demographic and socioeconomic characteristics including age (15-24, 25-64, $\geq 65$ years old), gender, living areas (rural or urban), level of education (primary school or below, middle/high school, college or above), occupation(student, unemployment, business and services, professionals, farmers and workers), household income ( $<100,000,100,000-199,999$ and $\geq 20,000$ yuan per year), children under 5 years old (yes or no), current smoking status (yes or no), and the history of common chronic diseases (yes or no).

\section{Statistical Analysis}

Socio-demographic characteristics of participants were described using frequency and percentage. Both univariate and multivariate logistic regression analyses were performed to estimate the associations of social-demographic characteristics and features of cough with health-seeking decision or choice of health facilities. All statistical analyses were conducted using SPSS 19.0 statistical software and all pvalues refer to two-tailed tests. Statistical significance was set at $\mathrm{P}<0.05$.

\section{Results}

\section{Characteristics of study population}


Of 5,855 eligible individuals interviewed, $69.6 \%$ of individuals aged $25-64$ years old, $10.8 \%$ were $\geq$ 65 years old, $50.7 \%$ were females and $40.0 \%$ living in rural areas. Around half had attended middle school, $22.7 \%$ graduated from college and above. 1,129 (19.3\%) reported coughing in the past month. Detailed characteristics of individuals are shown in Table 1.

\section{Factors associated with health-seeking decision}

Of the 1,129 participants who reported coughing in the past month, $40 \%$ (452) had been to medical facilities. The associations of socio-demographic characteristics and features of cough with healthseeking decision are presented in Table 2. Participants who aged $\geq 65$ years old, female and living in rural areas were associated 2.26 (95\% Cl: 1.24, 4.14), 1.57 (95\% Cl: 1.21, 2.07) and 1.31 (95\%Cl: 1.01, 1.70) times greater likelihood of seeking medical treatment respectively. Compared with individuals coughed for $\leq 2$ weeks, those coughed for $3-8$ weeks was 2.81 (95\% Cl: $1.68,4.73)$ times more likely to seek medical treatment. However, there was no significant difference between those coughed for more than 8 weeks and less than 2 weeks. A significant positive trend $(P<0.001)$ was observed between the number of accompanying symptoms and health-seeking decision. Compared with participants who reported no accompanying symptoms with cough, those reported symptoms were more likely to seek medical treatment, particularly for those with two symptoms and above (OR $=3.87,95 \% \mathrm{Cl}: 2.74,5.47)$.

\section{Factors associated with choice of medical facility}

Of the 452 participants who went for medical treatment, $120(26.5 \%)$ chose hospitals at county level or above. The associations of socio-demographic characteristics and features of cough with choice of medical facility are presented in Table 3. Female and people live in rural areas were $0.33(95 \% \mathrm{Cl}: 0.21$, $0.54)$ and 0.57 (95\% Cl: $0.36,0.92)$ times less likely to choose hospitals at county level or above respectively. Individuals with college degree or above were more likely to seek medical treatment in hospitals at or above the county level compared to those with education below primary school $(\mathrm{OR}=3.29$, $95 \% \mathrm{Cl}: 1.35,8.02)$. Participants who coughed for $3-8$ weeks and $>8$ weeks were associated with a higher likelihood of choosing hospitals at or above the county level $(\mathrm{OR}=2.35,95 \% \mathrm{Cl}: 1.19,4.61, \mathrm{OR}=3.13$, $95 \% \mathrm{Cl}$ : $1.24,7.90$, respectively). Number of accompanying symptoms does not show significant relationship with choosing medical facility. 
Table 1

Socio-demographic characteristics of the total population and individuals with cough in the past month.

\begin{tabular}{|c|c|c|c|c|}
\hline \multirow[t]{2}{*}{ Characteristics } & \multicolumn{2}{|c|}{ Total population } & \multicolumn{2}{|c|}{ Individuals with cough } \\
\hline & $\mathbf{N}$ & $\%$ & $\mathrm{~N}$ & $\%$ \\
\hline Total & 5855 & 100 & 1129 & 19.3 \\
\hline \multicolumn{5}{|l|}{ Age (years) } \\
\hline $15-24 y$ & 1146 & 19.6 & 245 & 21.4 \\
\hline $25-64 y$ & 4078 & 69.6 & 684 & 16.8 \\
\hline$\geq 65 y$ & 631 & 10.8 & 200 & 31.7 \\
\hline \multicolumn{5}{|l|}{ Residence } \\
\hline urban & 3512 & 60.0 & 671 & 19.1 \\
\hline rural & 2343 & 40.0 & 458 & 19.5 \\
\hline \multicolumn{5}{|l|}{ Gender } \\
\hline male & 2887 & 49.3 & 576 & 20 \\
\hline female & 2968 & 50.7 & 553 & 18.6 \\
\hline \multicolumn{5}{|l|}{ Occupation } \\
\hline student & 335 & 5.7 & 104 & 31 \\
\hline unemployed & 1461 & 25.0 & 337 & 23.1 \\
\hline Business/service & 2104 & 35.9 & 314 & 14.9 \\
\hline professional & 1247 & 21.3 & 232 & 18.6 \\
\hline Farmers and workers & 708 & 12.1 & 142 & 20.1 \\
\hline \multicolumn{5}{|l|}{ Education } \\
\hline Primary school and below & 1267 & 21.6 & 309 & 24.4 \\
\hline middle school & 3261 & 55.7 & 531 & 16.3 \\
\hline college and above & 1327 & 22.7 & 289 & 21.8 \\
\hline \multicolumn{5}{|l|}{ Child under 5 years old } \\
\hline No & 3450 & 58.9 & 708 & 20.5 \\
\hline Yes & 2405 & 41.1 & 421 & 17.5 \\
\hline
\end{tabular}

*Missing data for one individual. 


\begin{tabular}{|lllll|}
\hline Characteristics & \multicolumn{3}{c|}{ Total population } & \multicolumn{2}{l|}{ Individuals with cough } \\
\cline { 2 - 5 } & $\mathbf{N}$ & $\%$ & $\mathbf{N}$ & $\%$ \\
\hline Income (yuan)* & & & & \\
\hline$<100,000$ & 3372 & 57.6 & 667 & 19.8 \\
\hline $100,000-199,999$ & 1772 & 30.3 & 331 & 18.7 \\
\hline$\geq 200,000$ & 710 & 12.1 & 130 & 18.3 \\
\hline Smoking status* & & & & \\
\hline No & 3249 & 55.5 & 592 & 18.2 \\
\hline Yes & 2605 & 44.5 & 537 & 20.6 \\
\hline Chronic disease & & & & \\
\hline No & 4695 & 80.2 & 783 & 16.7 \\
\hline Yes & 1160 & 19.8 & 346 & 29.8 \\
\hline *Missing data for one individual. & & & \\
\hline
\end{tabular}


Table 2

Univariate and multivariate logistic regression analysis for health-seeking decision after coughing $(\mathrm{N}=$ $1,129)$

\begin{tabular}{|c|c|c|c|c|c|}
\hline \multirow[t]{2}{*}{ Characteristics } & \multirow{2}{*}{$\begin{array}{l}\text { Seeking } \\
\text { treatment } \\
\mathrm{N}(\%)\end{array}$} & \multicolumn{2}{|l|}{ Univariable } & \multicolumn{2}{|l|}{ Multivariable } \\
\hline & & Crude OR & $\begin{array}{l}P \\
\text { value }\end{array}$ & Adjusted OR & $\begin{array}{l}P \\
\text { value }\end{array}$ \\
\hline \multicolumn{6}{|l|}{ Age } \\
\hline $15-24 y$ & $86(35.1)$ & Ref & & Ref & \\
\hline $25-64 y$ & $267(39.0)$ & $\begin{array}{l}1.18 \\
(0.87,1.16)\end{array}$ & 0.277 & $\begin{array}{l}1.44 \\
(0.95,2.19)\end{array}$ & 0.089 \\
\hline$\geq 65 y$ & $99(49.5)$ & $\begin{array}{l}1.81 \\
(1.24,2.65)\end{array}$ & 0.002 & $\begin{array}{l}2.26 \\
(1.24,4.14)\end{array}$ & 0.008 \\
\hline \multicolumn{6}{|l|}{ Residence } \\
\hline urban & $247(36.8)$ & Ref & & Ref & \\
\hline rural & $205(44.8)$ & $\begin{array}{l}1.39 \\
(1.09,1.77)\end{array}$ & 0.008 & $\begin{array}{l}1.31 \\
(1.01,1.70)\end{array}$ & 0.044 \\
\hline \multicolumn{6}{|l|}{ Gender } \\
\hline male & $195(33.9)$ & Ref & & Ref & \\
\hline female & $257(46.5)$ & $\begin{array}{l}1.70 \\
(1.33,2.16)\end{array}$ & $<.001$ & $\begin{array}{l}1.57 \\
(1.21,2.07)\end{array}$ & 0.001 \\
\hline \multicolumn{6}{|l|}{ Occupation } \\
\hline student & $40(38.5)$ & Ref & & Ref & \\
\hline unemployed & $154(45.7)$ & $\begin{array}{l}1.35 \\
(0.86,2.11)\end{array}$ & 0.195 & $\begin{array}{l}1.18 \\
(0.61,2.29)\end{array}$ & 0.628 \\
\hline Business/service & $103(32.8)$ & $\begin{array}{l}0.78 \\
(0.49,1.24)\end{array}$ & 0.292 & $\begin{array}{l}0.98 \\
(0.52,1.84)\end{array}$ & 0.945 \\
\hline professional & $101(43.5)$ & $\begin{array}{l}1.23 \\
(0.77,1.98)\end{array}$ & 0.384 & $\begin{array}{l}1.34 \\
(0.71,2.53)\end{array}$ & 0.364 \\
\hline Farmers and workers & $54(38.0)$ & $\begin{array}{l}0.98 \\
(0.58,1.65)\end{array}$ & 0.945 & $\begin{array}{l}1.18 \\
(0.57,2.45)\end{array}$ & 0.654 \\
\hline \multicolumn{6}{|l|}{ Education } \\
\hline $\begin{array}{l}\text { Primary school and } \\
\text { below }\end{array}$ & $134(43.4)$ & Ref & & Ref & \\
\hline middle school & $225(38.7)$ & $\begin{array}{l}0.83 \\
(0.62,1.09)\end{array}$ & 0.179 & $\begin{array}{l}1.35 \\
(0.92,1.98)\end{array}$ & 0.127 \\
\hline
\end{tabular}




\begin{tabular}{|c|c|c|c|c|c|}
\hline \multirow[t]{2}{*}{ Characteristics } & \multirow{2}{*}{$\begin{array}{l}\text { Seeking } \\
\text { treatment } \\
\mathbf{N}(\%)\end{array}$} & \multicolumn{2}{|l|}{ Univariable } & \multicolumn{2}{|l|}{ Multivariable } \\
\hline & & Crude OR & $\begin{array}{l}P \\
\text { value }\end{array}$ & Adjusted OR & $\begin{array}{l}P \\
\text { value }\end{array}$ \\
\hline college and above & $93(38.9)$ & $\begin{array}{l}0.83 \\
(0.59,1.17)\end{array}$ & 0.294 & $\begin{array}{l}1.14 \\
(0.70,1.86)\end{array}$ & 0.605 \\
\hline \multicolumn{6}{|c|}{ Child under 5 years old } \\
\hline No & $290(41.0)$ & Ref & & & \\
\hline Yes & $162(38.5)$ & $\begin{array}{l}0.90 \\
(0.70,1.15)\end{array}$ & 0.411 & $\begin{array}{l}0.97 \\
(0.72,1.30)\end{array}$ & 0.829 \\
\hline \multicolumn{6}{|l|}{ Income (yuan) } \\
\hline$<100,000$ & $270(40.5)$ & Ref & & Ref & \\
\hline 100,000-199,999 & $134(40.5)$ & $\begin{array}{l}1.00 \\
(0.77,131)\end{array}$ & 0.999 & $\begin{array}{l}1.08 \\
(0.79,1.48)\end{array}$ & 0.644 \\
\hline$\geq 200,000$ & $47(36.2)$ & $\begin{array}{l}0.83 \\
(0.56,1.23)\end{array}$ & 0.357 & $\begin{array}{l}0.99 \\
(0.63,1.56)\end{array}$ & 0.968 \\
\hline \multicolumn{6}{|l|}{ Smoking status } \\
\hline No & $260(43.9)$ & $\begin{array}{l}1.41 \\
(1.11,1.79)\end{array}$ & 0.005 & $\begin{array}{l}1.23 \\
(0.94,1.60)\end{array}$ & 0.126 \\
\hline Yes & $192(35.8)$ & Ref & & Ref & \\
\hline \multicolumn{6}{|l|}{ Chronic disease } \\
\hline No & $285(36.4)$ & Ref & & Ref & \\
\hline Yes & $167(48.3)$ & $\begin{array}{l}1.63 \\
(1.26,2.11)\end{array}$ & $<.001$ & $\begin{array}{l}1.28 \\
(0.93,1.77)\end{array}$ & 0.128 \\
\hline \multicolumn{6}{|c|}{ Duration of cough(week) } \\
\hline$\leq 2$ & $372(37.5)$ & Ref & & Ref & \\
\hline $3-8$ & $56(68.3)$ & $\begin{array}{l}3.59 \\
(2.22,5.82)\end{array}$ & $\begin{array}{l}< \\
0.001\end{array}$ & $\begin{array}{l}2.81 \\
(1.68,4.73)\end{array}$ & $<0.001$ \\
\hline$>8$ & $24(43.6)$ & $\begin{array}{l}1.29 \\
(0.75,2.23)\end{array}$ & 0.362 & $\begin{array}{l}1.26 \\
(0.70,2.26)\end{array}$ & 0.448 \\
\hline \multicolumn{6}{|c|}{ Number of accompanying symptoms } \\
\hline 0 & $96(24.9)$ & Ref & & & \\
\hline 1 & $179(42.1)$ & $\begin{array}{l}2.19 \\
(1.62,2.96)\end{array}$ & $<0.001$ & $\begin{array}{l}2.19 \\
(1.59,3.01)\end{array}$ & $<.001$ \\
\hline
\end{tabular}




\begin{tabular}{|llllll|}
\hline Characteristics & $\begin{array}{l}\text { Seeking } \\
\text { treatment }\end{array}$ & Univariable & \multicolumn{3}{c|}{ Multivariable } \\
\cline { 3 - 6 } & $\mathbf{N}(\%)$ & Crude OR & $\begin{array}{l}\mathbf{P} \\
\text { value }\end{array}$ & Adjusted OR & $\begin{array}{l}\mathbf{P} \\
\text { value }\end{array}$ \\
\hline$\geq 2$ & $177(55.5)$ & $\mathbf{3 . 7 5}$ & $<$ & $\mathbf{3 . 8 7}$ & $<$ \\
& & $(2.73,5.17)$ & 0.001 & $\mathbf{( 2 . 7 4 , 5 . 4 7 )}$ & 0.001 \\
\hline
\end{tabular}


Table 3

Univariate and multivariate logistic regression analysis for choice of medical facility after coughing $(\mathrm{N}=$ 452)

\begin{tabular}{|c|c|c|c|c|c|c|}
\hline \multirow[t]{2}{*}{ Characteristics } & \multicolumn{2}{|c|}{ Choice of Medical Facility } & \multirow[t]{2}{*}{ Crude OR } & \multirow{2}{*}{$\begin{array}{l}P \\
\text { value }\end{array}$} & \multirow{2}{*}{$\begin{array}{l}\text { Adjusted } \\
\text { OR }\end{array}$} & \multirow{2}{*}{$\begin{array}{l}P \\
\text { value }\end{array}$} \\
\hline & $\begin{array}{l}\text { Community } \\
\text { health service } \\
\text { center and } \\
\text { below } \mathrm{N}(\%)\end{array}$ & $\begin{array}{l}\text { hospital at } \\
\text { county level } \\
\text { and above N } \\
(\%)\end{array}$ & & & & \\
\hline \multicolumn{7}{|l|}{ Age } \\
\hline $15-24 y$ & $61(70.9)$ & $25(29.1)$ & Ref & & Ref & \\
\hline $25-64 y$ & $207(77.5)$ & $60(22.5)$ & $\begin{array}{l}0.71 \\
(0.41,1.22)\end{array}$ & 0.215 & $\begin{array}{l}0.84 \\
(0.39,1.81)\end{array}$ & 0.649 \\
\hline$\geq 65 y$ & $64(64.6)$ & $35(35.4)$ & $\begin{array}{l}1.33 \\
(0.72,2.49)\end{array}$ & 0.363 & $\begin{array}{l}2.72 \\
(0.90,8.20)\end{array}$ & 0.075 \\
\hline \multicolumn{7}{|l|}{ Residence } \\
\hline urban & $162(65.6)$ & $85(34.4)$ & Ref & & Ref & \\
\hline rural & $170(82.9)$ & $35(17.1)$ & $\begin{array}{l}0.39 \\
(0.25,0.62)\end{array}$ & <. 001 & $\begin{array}{l}0.33 \\
(0.21,0.54)\end{array}$ & $\begin{array}{l}<.001 \\
0.00\end{array}$ \\
\hline \multicolumn{7}{|l|}{ Gender } \\
\hline male & $135(69.2)$ & $60(30.8)$ & Ref & & Ref & \\
\hline female & 197 (76.7) & $60(23.3)$ & $\begin{array}{l}0.69 \\
(0.45,1.04)\end{array}$ & 0.077 & $\begin{array}{l}0.57 \\
(0.36,0.92)\end{array}$ & 0.021 \\
\hline \multicolumn{7}{|l|}{ Occupation } \\
\hline student & $27(67.5)$ & $13(32.5)$ & Ref & & Ref & \\
\hline unemployed & $112(72.7)$ & $42(27.3)$ & $\begin{array}{l}0.78 \\
(0.37,1.65)\end{array}$ & 0.514 & $\begin{array}{l}0.73 \\
(0.22,2.41)\end{array}$ & 0.61 \\
\hline $\begin{array}{l}\text { Business/service } \\
\text { personnel }\end{array}$ & $76(73.8)$ & $27(26.2)$ & $\begin{array}{l}0.74 \\
(0.33,1.63)\end{array}$ & 0.453 & $\begin{array}{l}0.65 \\
(0.21,2.04)\end{array}$ & 0.457 \\
\hline professional & $78(77.2)$ & $23(22.8)$ & $\begin{array}{l}0.61 \\
(0.27,1.38)\end{array}$ & 0.235 & $\begin{array}{l}0.43 \\
(0.14,1.31)\end{array}$ & 0.137 \\
\hline $\begin{array}{l}\text { Farmers and } \\
\text { workers }\end{array}$ & $39(72.2)$ & $15(27.8)$ & $\begin{array}{l}0.80 \\
(0.33,1.95)\end{array}$ & 0.621 & $\begin{array}{l}0.74 \\
(0.20,2.80)\end{array}$ & 0.659 \\
\hline \multicolumn{7}{|l|}{ Education } \\
\hline $\begin{array}{l}\text { Primary school } \\
\text { and below }\end{array}$ & $99(73.9)$ & $35(26.1)$ & Ref & & Ref & \\
\hline
\end{tabular}




\begin{tabular}{|c|c|c|c|c|c|c|}
\hline \multirow[t]{2}{*}{ Characteristics } & \multicolumn{2}{|c|}{ Choice of Medical Facility } & \multirow[t]{2}{*}{ Crude OR } & \multirow{2}{*}{$\begin{array}{l}P \\
\text { value }\end{array}$} & \multirow{2}{*}{$\begin{array}{l}\text { Adjusted } \\
\text { OR }\end{array}$} & \multirow{2}{*}{$\begin{array}{l}\mathrm{P} \\
\text { value }\end{array}$} \\
\hline & $\begin{array}{l}\text { Community } \\
\text { health service } \\
\text { center and } \\
\text { below } \mathbf{N}(\%)\end{array}$ & $\begin{array}{l}\text { hospital at } \\
\text { county level } \\
\text { and above N } \\
(\%)\end{array}$ & & & & \\
\hline middle school & $171(76)$ & $54(24)$ & $\begin{array}{l}0.89 \\
(0.55,1.46)\end{array}$ & 0.653 & $\begin{array}{l}1.44 \\
(0.69,3.01)\end{array}$ & 0.332 \\
\hline $\begin{array}{l}\text { college and } \\
\text { above }\end{array}$ & $62(66.7)$ & 31 (33.3) & $\begin{array}{l}1.41 \\
(0.79,2.52)\end{array}$ & 0.24 & $\begin{array}{l}3.29 \\
(1.35,8.02)\end{array}$ & 0.009 \\
\hline \multicolumn{7}{|l|}{ Child under 5} \\
\hline no & $206(71)$ & $84(29)$ & Ref & & Ref & \\
\hline yes & $126(77.8)$ & $36(22.2)$ & $\begin{array}{l}0.70 \\
(0.45,1.10)\end{array}$ & 0.12 & $\begin{array}{l}0.75 \\
(0.42,1.34)\end{array}$ & 0.33 \\
\hline \multicolumn{7}{|l|}{$\begin{array}{l}\text { Household } \\
\text { income(yuan) }\end{array}$} \\
\hline$<100,000$ & $203(75.2)$ & $67(24.8)$ & Ref & & Ref & \\
\hline $\begin{array}{l}100,000- \\
199,999\end{array}$ & $100(74.6)$ & $34(25.4)$ & $\begin{array}{l}1.03 \\
(0.64,1.66)\end{array}$ & 0.903 & $\begin{array}{l}0.98 \\
(0.55,1.75)\end{array}$ & 0.937 \\
\hline$\geq 200,000$ & $28(59.6)$ & $19(40.4)$ & $\begin{array}{l}2.06 \\
(1.08,3.92)\end{array}$ & 0.028 & $\begin{array}{l}3.29 \\
(1.35,8.02)\end{array}$ & 0.161 \\
\hline \multicolumn{7}{|l|}{ Smoking status } \\
\hline yes & 148 (77.1) & $44(22.9)$ & Ref & & Ref & \\
\hline no & $184(70.8)$ & $76(29.2)$ & $\begin{array}{l}1.39 \\
(0.90,2.14)\end{array}$ & 0.134 & $\begin{array}{l}1.38 \\
(0.85,2.24)\end{array}$ & 0.193 \\
\hline \multicolumn{7}{|l|}{ Chronic disease } \\
\hline no & $210(73.7)$ & $75(26.3)$ & Ref & & Ref & \\
\hline yes & $122(73.1)$ & $45(26.9)$ & $\begin{array}{l}1.03 \\
(0.67,1.59)\end{array}$ & 0.884 & $\begin{array}{l}0.63 \\
(0.35,1.14)\end{array}$ & 0.123 \\
\hline \multicolumn{7}{|l|}{$\begin{array}{l}\text { Duration of } \\
\text { cough(week) }\end{array}$} \\
\hline$\leq 2$ & $284(76.3)$ & $88(23.7)$ & Ref & & Ref & \\
\hline $3-8$ & $35(62.5)$ & $21(37.5)$ & $\begin{array}{l}1.94 \\
(1.07,3.50)\end{array}$ & 0.029 & $\begin{array}{l}2.35 \\
(1.19,4.61)\end{array}$ & 0.014 \\
\hline$>8$ & $13(54.2)$ & $11(45.8)$ & $\begin{array}{l}2.73 \\
(1.18,6.31)\end{array}$ & 0.019 & $\begin{array}{l}3.13 \\
(1.24,7.90)\end{array}$ & 0.016 \\
\hline
\end{tabular}




\begin{tabular}{|c|c|c|c|c|c|c|}
\hline \multirow[t]{2}{*}{ Characteristics } & \multicolumn{2}{|c|}{ Choice of Medical Facility } & \multirow[t]{2}{*}{ Crude OR } & \multirow{2}{*}{$\begin{array}{l}P \\
\text { value }\end{array}$} & \multirow{2}{*}{$\begin{array}{l}\text { Adjusted } \\
\text { OR }\end{array}$} & \multirow{2}{*}{$\begin{array}{l}\mathrm{P} \\
\text { value }\end{array}$} \\
\hline & $\begin{array}{l}\text { Community } \\
\text { health service } \\
\text { center and } \\
\text { below N (\%) }\end{array}$ & $\begin{array}{l}\text { hospital at } \\
\text { county level } \\
\text { and above N } \\
\text { (\%) }\end{array}$ & & & & \\
\hline \multicolumn{7}{|l|}{$\begin{array}{l}\text { Number of } \\
\text { accompanying } \\
\text { symptoms }\end{array}$} \\
\hline 0 & $69(71.9)$ & $27(28.1)$ & Ref & & Ref & \\
\hline 1 & $131(73.2)$ & $48(26.8)$ & $\begin{array}{l}0.94 \\
(0.54,1.63)\end{array}$ & 0.816 & $\begin{array}{l}1.25 \\
(0.66,2.36)\end{array}$ & 0.502 \\
\hline$\geq 2$ & $132(74.6)$ & $45(25.4)$ & $\begin{array}{l}0.87 \\
(0.50,1.52)\end{array}$ & 0.629 & $\begin{array}{l}1.14 \\
(0.59,2.18)\end{array}$ & 0.704 \\
\hline
\end{tabular}

\section{Discussion}

To the best of our knowledge, the present study is the first community-based study focused on the association of socio-demographic and cough characteristics with health seeking behavior in the Chinese population. We found that $19.3 \%$ of the respondents reported a cough in the past month, $40 \%$ of them had sought medical treatment. Individuals more than 65 years old, female, with cough for 3-8 weeks and with more accompanying symptoms were more likely to seek medical treatment. For those had sought medical treatment, $26.5 \%$ of them chose hospitals at county level or above. Male, people live in urban areas, individuals graduated from college and above and with cough for more than 2 weeks were more likely to choose hospitals at county level or above.

\section{Health-seeking decision}

Contrary to some previous studies on health-seeking behavior ${ }^{[12,14,15]}$, we found that some of the sociodemographic characteristics, such as education, occupation, family size and household income do not seem to be associated with the probability of seeking medical treatment after coughing. Smoking status and history of chronic disease were also not significantly associated with health-seeking decision after adjusting for other factors. These may be related to population selection, and heterogeneity in different countries.

The present study found that individuals aged $\geq 65$ years old and living in rural areas were more likely to seek medical treatment. Besides, women were more likely to seek medical treatment than men, which is in parallel with studies conducted in Zambia and Vietnam ${ }^{[15,24]}$. This may be explained by the fact that women were more troubled by cough than men ${ }^{[25,26]}$. What's more, some studies focusing on gender differences in health-seeking behavior have pointed out that men were more likely to behave masculinity and a healthy state thus less likely to seek care ${ }^{[27]}$. 
The present study was the first to focus on the association between cough duration and health-seeking decision in China. It is highly worth noting that individuals with cough for 3-8 weeks were more likely to seek medical treatment, while those coughed for $>8$ weeks were not. The possible explanation for this phenomenon could be that individuals who have been coughing for $>8$ weeks were already used to coughing and regard it as a lifestyle habit rather than a disease. However, in addition to common causes of chronic cough, recent studies have found that pertussis has became a prevalent disease in some areas $[7,8]$, and B. pertussis infection should be considered as a significant pathogenic infection in adult patients presenting a cough of more than 3 weeks duration ${ }^{[28,29]}$. And tuberculosis should be evaluated also among persons with unexplained productive cough lasting 2-3 weeks or more ${ }^{[6]}$. Thus there is no doubt that prolonged coughing without seeking medical treatment timely will bring an increased risk of transmission of such respiratory infectious disease. It is extremely desirable to undertake extensive educational campaigns about cough especially persistent one, so as to encourage individuals to visit medical facilities in time.

What's more, the number of accompanying symptoms showed a clear increasing trend with healthseeking decision, as were observed in India and Tanzania ${ }^{[13,20]}$. Previous studies have also indicated that if a cough is not distressing or associated with any other symptoms that restrict one's ability to function/work, people do not usually seek medical care ${ }^{[30,31]}$. Because the cough often be regarded as very common symptom and not an illness, whereas cough together with other symptoms, such as fever, expectoration, is more consistently felt to be an illness.

\section{Choice of medical facility}

In the present study, the multivariate analysis consistently show that individuals living in rural areas and women were less likely to choose hospitals at or above the county level, while the most educated persons were more likely. Studies conducted in Vietnam ${ }^{[24]}$ and China ${ }^{[23,32]}$ also found that women took more health-care actions than men, but chose less qualified providers (like self-medication, pharmacist, or private practitioner) and individuals living in rural areas had poor access to medical facility. But contrary to previous studies conducted in China ${ }^{[21,22,33]}$, the household income was not significant associated with the choice of medical facility after adjusting for other confounders in the present study. This change may be due to a decline in health spending variance as the economy develops, with rising living standards and incomes.

We also found that individuals who coughed for more than 2 weeks were associated with a higher likelihood of choosing hospitals at or above the county level, while number of accompanying symptoms did not show any significant relationship with their choice. This reveals an interesting fact that people were more likely to seek medical treatment when the accompany symptoms of cough appear, but pay more attention to the duration of cough when choosing a hospital. The result echoes a call in a Japanese study ${ }^{[34]}$, which suggested that individuals suffering from cough that does not resolve within a short period of time should be taken seriously regardless of the severity, as cough can be a sign of serious 
diseases such as lung cancer, pertussis and pulmonary tuberculosis ${ }^{[2,35,36]}$. Based on the fact, it is recommended to strengthen the training of relevant personnel in such facilities to improve their attention to persistent cough, as well as the level of surveillance and case detection of the above serious diseases.

\section{Limitations}

Although the data in the present study was based on a community-based population and corrected for established and potential confounding factors (both socio-demographic and cough characteristics), these findings should also be interpreted in light of some limitations. First, it is important to acknowledge the limitations of the subjective perceptions and self-reports and therefore may be biased by potential under-reporting or over-reporting and recall bias, even though we asked for information within the past month to reduce recall bias. Second, due to the limited sample size, some significant positive associations may not be demonstrated in the present study. Finally, the study cannot exclude the effects of residual confounding by unmeasured risk factors, such as medical insurance and cause of cough. Therefore, larger sample size studies with more adjusted confounders are warranted to examine the further association and make a firm complement to the current study.

\section{Conclusion}

The present study found that only a small proportion of individuals sought medical treatment after cough and of these, about $1 / 3$ chose hospitals at county level or above. Age, gender, living areas, education level as well as features of cough were associated with health seeking behavior. Targeted intervention measures should be formulated based on the above key factors to guide people to seek medical treatment scientifically and rationally.

\section{Declarations}

\section{Ethics approval and consent to participate}

The Ethical Review Committee of the Chinese Center for Disease Control and Prevention (Beijing, China) approved the study. All the participants provided written informed consent.

\section{Consent for publication}

Not applicable.

\section{Availability of data and materials}

The datasets used and/or analyzed during the current study are available from the corresponding author on reasonable request. 


\section{Competing interests}

The authors declare that they have no competing interest.

\section{Funding}

The work was supported by the following grants: National Natural Science Foundation of China (NSFC) [grant number 81973106], Beijing Municipal Natural Science Foundation [grant number 19L2060].

\section{Authors' contributions}

Xiaoyan Sun, Lingqiao Lou, Hang Cheng, Zhen Ye, Jianwei Jia, Yina Wei, Jingbo Tao performed data collection, collation and preliminary analysis. Xiaoyan Sun and Shuying Luo performed data analysis, interpreted results, participated in writing and editing the manuscript, and Hanqing He helped revise manuscript. All authors read and approved the final manuscript.

\section{Acknowledgements}

We sincerely thank Dr. Jianxin Yu (Chinese Center for Disease Control and Prevention) for his help to design this study.

\section{Author details}

${ }^{1}$ Yiwu Center for Disease Control and Prevention, Yiwu, 322000, P.R. China

${ }^{2}$ Zhejiang provincial Center for Disease Control and Prevention, Hangzhou 310051, P.R. China

\section{References}

1. Bulgiba AM, Razaz M. How well can signs and symptoms predict AMI in the Malaysian population? International journal of cardiology 2005, 102(1):87-93.

2. F H, S B, L D et al. The diagnosis and treatment of acute cough in adults. Deutsches Arzteblatt international 2014, 111(20):356-363.

3. Cho SH, Lin HC, Ghoshal AG et al. Respiratory disease in the Asia-Pacific region: Cough as a key symptom. Allergy and asthma proceedings 2016, 37(2):131-140.

4. Edwards KM. Is pertussis a frequent cause of cough in adolescents and adults? Should routine pertussis immunization be recommended? Clinical infectious diseases 2001, 32(12):1698-1699. 
5. WHO Guideline Development Group. WHO Guidelines Approved by the Guidelines Review Committee[J]. Treatment of Tuberculosis: Guidelines 2010.

6. Hopewell PC, Pai M, Maher D et al. International standards for tuberculosis care. The Lancet Infectious diseases 2006, 6(11):710-725.

7. Hu JJ, Lu CY, Chang LY et al. Survey of pertussis in patients with prolonged cough[J]. J Microbiol Immunol Infect, 2006, 39(1):54-58.

8. Dalby T, Harboe ZB, Krogfelt KA. Seroprevalence of pertussis among Danish patients with cough of unknown etiology. Clinical and vaccine immunology 2010, 17(12):2016-2023.

9. Lee SY, Han SB, Kang JH et al. Pertussis Prevalence in Korean Adolescents and Adults with Persistent Cough. Journal of Korean medical science 2015, 30(7):988-990.

10. K L, J P, R C et al. Epidemiology of cough in relation to China. Cough 2013, 9(1):18.

11. Lai K LB, Wang F, Chen R, Liu X, Zhong N. Survey on the diagnosis and management of the patients with chronic cough. Int J Respir 2011, 31:645-647.

12. Christian $\mathrm{C}$, Burger $\mathrm{C}$, Claassens $\mathrm{M}$ et al. Patient predictors of health-seeking behaviour for persons coughing for more than two weeks in high-burden tuberculosis communities: the case of the Western Cape, South Africa. BMC health services research 2019, 19(1):160.

13. Senkoro M, Hinderaker SG, Mfinanga SG et al. Health care-seeking behaviour among people with cough in Tanzania: findings from a tuberculosis prevalence survey. The international journal of tuberculosis and lung disease 2015, 19(6):640-646.

14. Senbeto $M$, Tadesse $S$, Tadesse $T$ et al. Appropriate health-seeking behavior and associated factors among people who had cough for at least two weeks in northwest Ethiopia: a population-based cross-sectional study. BMC public health 2013, 13:1222.

15. Chanda-Kapata P, Kapata N, Masiye F et al. Health Seeking Behaviour among Individuals with Presumptive Tuberculosis in Zambia. PloS one 2016, 11(10):e0163975.

16. Satyanarayana S, Nair SA, Chadha SS et al. Health-care seeking among people with cough of 2 weeks or more in India. Is passive TB case finding sufficient? Public Health Action 2012, 2(4):157161.

17. Godfrey-Faussett $P$, Kaunda $H$, Kamanga $J$ et al. Why do patients with a cough delay seeking care at Lusaka urban health centres? A health systems research approach. The international journal of tuberculosis and lung disease 2002, 6(9):796-805.

18. Addisu Y, Birhanu Z, Tilahun D et al. Predictors of treatment seeking intention among people with cough in East Wollega, Ethiopia based on the theory of planned behavior: a community based crosssectional study. Ethiopian journal of health sciences 2014, 24(2):131-138.

19. Fei Y, Wang JM, Zhang JH et al. Access to tuberculosis care among community patients with chronic cough in Yangzhong County, Jiangsu Province. Journal of hygiene research 2006, 35(2):155-158.

20. Fochsen G, Deshpande K, Diwan V et al. Health care seeking among individuals with cough and tuberculosis: a population-based study from rural India. The international journal of tuberculosis and 
lung disease 2006, 10(9):995-1000.

21. Yi Hu, Biao Xu, Qi Zhao et al. Health seeking behaviors and expenditures for chronic cough in outpatients of county general hospitals in northern rural Jiangsu province. Fudan Univ J Med Sci 2006(01):39-43.

22. Qingwu Jiang, Biao Xu, Jie Tang et al. Analysis of medical-seeking behavior and influencing factors of patients with chronic cough in Jiangsu rural areas. Supplement to Volume 25 of 2003 Chinese Journal of Antituberculosis-2003 National Academic Conference of China National Antituberculosis Association Proceedings. 2003.

23. Weiying Liu, Qin Yu, Hongmei Yue, et al. The Gender Difference and Hospitalizing Behavior of Pafients with Chronic Cough in Lanzhou. Chinese Primary Health Care, 2017, 31(10)凶37.

24. Thorson A, Hoa NP, Long NH: Health-seeking behaviour of individuals with a cough of more than 3 weeks. Lancet 2000, 356(9244):1823-1824.

25. French CT, Fletcher KE, Irwin RS: Gender differences in health-related quality of life in patients complaining of chronic cough. Chest 2004, 125(2):482-488.

26. CT F, KE F, RS I: A comparison of gender differences in health-related quality of life in acute and chronic coughers. Chest 2005, 127(6):1991-1998.

27. Chikovore J, Hart G, Kumwenda $M$ et al. Control, struggle, and emergent masculinities: a qualitative study of men's care-seeking determinants for chronic cough and tuberculosis symptoms in Blantyre, Malawi. BMC public health 2014, 14:1053.

28. Kapaskelis AM, Vouloumanou EK, Rafailidis PI et al. High prevalence of antibody titers against Bordetella pertussis in an adult population with prolonged cough. Respiratory medicine 2008, 102(11):1586-1591.

29. Senzilet L D , Halperin S A , Spika J S , et al. Pertussis Is a Frequent Cause of Prolonged Cough Illness in Adults and Adolescents[J]. Clinical Infectious Diseases(12):1691-1697.

30. Banerji D, Andersen S. A sociological study of awareness of symptoms among persons with pulmonary tuberculosis. Bulletin of the World Health Organization 1963, 29(5):665-683.

31. Blaiss MS, Dicpinigaitis PV, Eccles R et al. Consumer attitudes on cough and cold: US (ACHOO) survey results. Current medical research and opinion 2015, 31(8):1527-1538.

32. Dongmei Liu, Yang Fei, Qunhui Ren et al. Gender differences in Health Seeking Behaviour and Tuberculosis Care Access of Potential TB Patients. Chinese Primary Health Care, 2006, 20(10): 5860

33. Biao Xu, Yang Fei, Qi Zhao. The impact of medical treatment of patients with chronic cough in rural communities on the detection of tuberculosis patients. National Academic Conference of Chinese Antituberculosis Association. 2007.

34. Fujimura M: Frequency of persistent cough and trends in seeking medical care and treatment-results of an internet survey. Allergology international 2012, 61(4):573-581. 
35. Moore A, Ashdown HF, Shinkins B et al. Clinical Characteristics of Pertussis-Associated Cough in Adults and Children: A Diagnostic Systematic Review and Meta-Analysis. Chest 2017, 152(2):353367.

36. Assefa Y, Woldeyohannes S, Gelaw YA et al. Screening tools to exclude active pulmonary TB in high TB burden countries: systematic review and meta-analysis. The international journal of tuberculosis and lung disease 2019, 23(6):728-734. 